\title{
Thyroid Gland Leiomyosarcoma
}

National Cancer Institute

\section{Source}

National Cancer Institute. Thyroid Gland Leiomyosarcoma. NCI Thesaurus. Code C156347.

A leiomyosarcoma that arises from the thyroid gland. 